\title{
Revisiting the Temkin Isotherm: Dimensional Inconsistency and Approximate Forms
}

\author{
Khim Hoong Chu
}

Honeychem Research, Newtown, Wellington 6021, New Zealand

E-mail: khimchu@gmail.com

Industrial \& Engineering Chemistry Research DOI: https://doi.org/10.1021/acs.iecr.1c01788

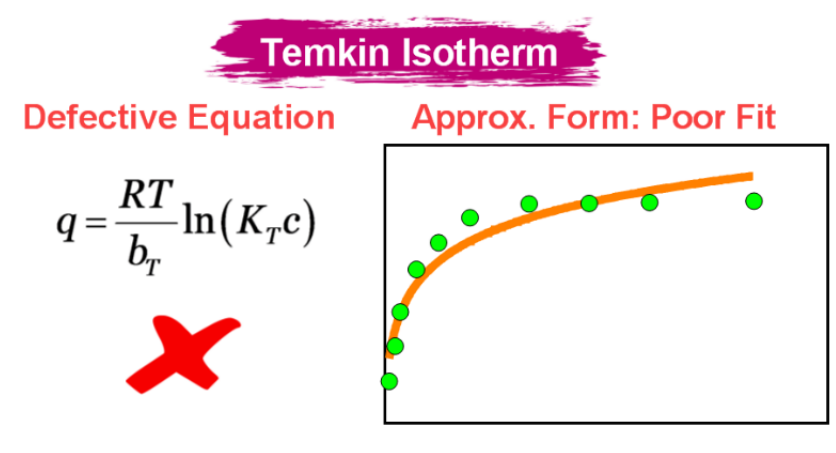

\begin{abstract}
The Temkin isotherm equation is increasingly being used to correlate adsorption isotherms of water contaminants. This research note shows that the Temkin isotherm as used in environmental adsorption research suffers from a fundamental flaw of dimensional inconsistency. In addition, an important but frequently overlooked issue concerns the approximate nature of the Temkin isotherm which limits its ability to fit type I isotherms: it has neither a Henry's law limit at low loadings nor a finite saturation limit at high loadings. A different approximate Temkin isotherm, which approaches a linear isotherm at low concentrations and commences at the $(0,0)$ origin, is shown to outperform the commonly used Temkin isotherm in terms of fitting ability and resembles the Langmuir and Freundlich isotherms in describing adsorption data without a saturation plateau. It is hoped that the material presented in this research note will lead to a better comprehension of the Temkin isotherm.
\end{abstract}




\section{INTRODUCTION}

Recent research on the use of porous materials to remove a diverse array of organic and inorganic water contaminants has generated vast literature. ${ }^{1}$ For any chosen contaminantadsorbent system, an essential topic of investigation involves experimental determinations of adsorption equilibrium and kinetics. In practice, equilibrium data are collected by suspending the adsorbent particles in a contaminant solution and allowing sufficient time for equilibrium to be attained. The measured equilibrium data, commonly presented as isotherms showing the solid-phase concentration plotted against the solution-phase concentration, are generally correlated using suitable theoretical or empirical isotherm models.

Table 1. Use of Temkin and Other Isotherms in Some Recent Papers Published in Industrial \& Engineering Chemistry Research ${ }^{\mathrm{a}}$

\begin{tabular}{|c|c|c|}
\hline reference & contaminant & isotherm \\
\hline Liu et al. ${ }^{2}$ & uranium & $\mathrm{F}, \mathrm{L}, \mathrm{T}$ \\
\hline Ashrafi et al. ${ }^{3}$ & methylene blue & DR, F, L, T \\
\hline Du et al. ${ }^{4}$ & mercury & $\mathrm{F}, \mathrm{L}, \mathrm{T}$ \\
\hline Xie et al. ${ }^{5}$ & methylene blue & $\mathrm{DR}, \mathrm{E}, \mathrm{H}, \mathrm{HJ}, \mathrm{F}, \mathrm{L}, \mathrm{T}$ \\
\hline Ouyang et al. ${ }^{6}$ & arsenic & $\mathrm{F}, \mathrm{L}, \mathrm{T}, \mathrm{RP}$ \\
\hline Hao et al. ${ }^{7}$ & cadmium & $\mathrm{DR}, \mathrm{F}, \mathrm{L}, \mathrm{LF}, \mathrm{RP}, \mathrm{T}$ \\
\hline Elmi et al. ${ }^{8}$ & metribuzin & $\mathrm{DR}, \mathrm{F}, \mathrm{L}, \mathrm{T}$ \\
\hline Afshari et al. ${ }^{9}$ & mercury & $\mathrm{F}, \mathrm{L}, \mathrm{T}$ \\
\hline Guo et al. ${ }^{10}$ & dyes & $\mathrm{F}, \mathrm{L}, \mathrm{T}$ \\
\hline Zheng et al. ${ }^{11}$ & chromium & HL, L, T \\
\hline Trujillo et al. $^{12}$ & dyes & $\mathrm{F}, \mathrm{L}, \mathrm{T}$ \\
\hline Sivalingam and $\operatorname{Sen}^{13}$ & arsenic & $\mathrm{DR}, \mathrm{F}, \mathrm{L}, \mathrm{T}$ \\
\hline Huang and Wang ${ }^{14}$ & lithium & $\mathrm{F}, \mathrm{L}, \mathrm{T}$ \\
\hline Ge et al. ${ }^{15}$ & iopamidol & $\mathrm{E}, \mathrm{F}, \mathrm{L}, \mathrm{T}$ \\
\hline
\end{tabular}

aDR: Dubinin-Radushkevich; E: Elovich; F: Freundlich; H: Halsey; HJ: Harkins-Jura; HL: Henry's law; L: Langmuir; LF: Langmuir-Freundlich; RP: Redlich-Peterson; T: Temkin

The bulk of observed equilibrium isotherms for aqueous systems are of type I form in Brunauer's classification, which manifest a monotonic approach to a limiting adsorption capacity. Several isotherm equations are available to describe such curve shapes, one of which is the Temkin isotherm which has in recent years been used in an increasing number of articles reporting on water contaminant adsorption. Table 1 presents a list of such papers published 
recently in Industrial \& Engineering Chemistry Research. ${ }^{2-15}$ One observation that jumps out from Table 1 is that the data fitting ability of the Temkin isotherm is often compared with those of the Langmuir and Freundlich isotherms. Although not as popular as the latter two isotherms, the Temkin isotherm is being used with sufficient frequency to merit wider awareness of its strengths and limitations. In this research note, we revisit the Temkin isotherm to highlight some critical issues not frequently recognized by environmental adsorption researchers.

A common form of the Temkin isotherm as used in environmental adsorption research can be written as eq 1 where $q$ is the solid-phase concentration, $c$ is the equilibrium liquidphase concentration, $R$ is the gas constant, $T$ is the temperature, and $b_{\mathrm{T}}$ and $K_{\mathrm{T}}$ are adjustable parameters.

$q=\frac{R T}{b_{\mathrm{T}}} \ln \left(K_{\mathrm{T}} c\right)$

In some publications the Temkin isotherm defined by eq 1 is referred to as the Tempkin isotherm. ${ }^{16}$ Since the Temkin isotherm is of the correct functional form to represent a type I isotherm, it may be used to fit typical experimental isotherms of water contaminants by appropriate choice of the constants $b_{\mathrm{T}}$ and $K_{\mathrm{T}}$. To fit eq 1 to a type $\mathrm{I}$ isotherm by a linear regression procedure, it is usually rewritten as eq 2. If this equation is obeyed, plots of $q$ against $\ln (c)$ should give a straight line.

$q=\frac{R T}{b_{\mathrm{T}}} \ln \left(K_{\mathrm{T}}\right)+\frac{R T}{b_{\mathrm{T}}} \ln (c)$

There are however two critical issues concerning the Temkin isotherm that seem to have attracted hardly any attention. The first is that eq 1 is a dimensionally inconsistent formulation. The second issue concerns the range of validity of eq 1: it is an approximate Temkin equation valid for intermediate $q$ values. Therefore, eq 1 should not be used to describe the entire range of an observed isotherm profile. Because this paper introduces a different approximate Temkin equation, to avoid confusion, eq. 1 (and its dimensionally consistent 
counterpart) will hereafter be called the Temkin-I equation. Several reviews ${ }^{17-21}$ have featured the Temkin-I equation but nothing is said about the two issues mentioned above. Two very recent reviews ${ }^{22,23}$ point out the restrictive condition of use but do not address the dimensional inconsistency problem. As such, the primary intent of this research note is to spotlight the two important but generally unrecognized issues pertaining to the Temkin-I equation. The secondary aims are to (1) present the complete Temkin isotherm, (2) introduce a more useful approximate Temkin equation, which is called the Temkin-II equation, and (3) use simulated data to compare the Temkin-II, Langmuir, and Freundlich equations. Literature isotherm data are used in some cases to illustrate the key points of this work. 